\title{
Existence of least energy nodal solution for a Schrödinger-Poisson system in bounded domains*
}

\author{
Claudianor O. Alves ${ }^{\dagger}$ and Marco A.S. Souto \\ Universidade Federal de Campina Grande \\ Unidade Acadêmica de Matemática e Estatística \\ CEP:58429-900, Campina Grande - PB, Brazil.
}

2000 Mathematics Subject Classification:35J20, 35J65

Keywords: Schrödinger-Poisson systems, nodal solution, variational methods

\begin{abstract}
We prove the existence of least energy nodal solution for a class of Schrödinger-Poisson system in a bounded domain $\Omega \subset \mathbb{R}^{3}$ with nonlinearity having a subcritical growth.
\end{abstract}

\section{Introduction}

This paper was motivated by some works that have appeared in recent years concerning with the nonlinear Schrödinger-Poisson system

$$
\left\{\begin{array}{l}
-i \frac{\partial \psi}{\partial t}=-\Delta \psi+\phi(x) \psi-|\psi|^{p-2} \psi \text { in } \Omega \\
-\Delta \phi=|\psi|^{2} \text { in } \Omega \\
\phi=\psi=0 \text { on } \partial \Omega
\end{array}\right.
$$

where $\Omega \subset \mathbb{R}^{3}$ is a bounded domain with smooth boundary, $2<p<2^{*}=6$ and $\psi: \bar{\Omega} \rightarrow \mathbb{C}$ and $\phi: \bar{\Omega} \rightarrow \mathbb{R}$ are unknown functions.

*Partially supported by INCT-MAT, Casadinho/PROCAD 552464/2011-2

${ }^{\dagger}$ C.O. Alves was partially supported by CNPq/Brazil 303080/2009-4, coalves@dme.ufcg.edu.br

${ }_{\ddagger}^{\ddagger}$ M.A.S. Souto was supported by CNPq/Brazil 304652/2011-3, marco@dme.ufcg.edu.br 
The first equation in $(N S P)$, called Schrödinger equation, describes quantum (non-relativistic) particles interacting with the eletromagnetic field generated by the motion. An interesting Schrödinger equation class is when the potential $\phi(x)$ is determined by the charge of wave function itself, that is, when the second equation in $(N S P)$ (Poisson equation) holds.

Knowledge of the solutions for the elliptic equation

$$
\left\{\begin{array}{l}
-\Delta u+\phi u=f(u) \text { in } \Omega, \\
-\Delta \phi=u^{2} \text { in } \Omega, \\
u, \phi=0, \partial \Omega
\end{array}\right.
$$

has a great importance in the study of stationary solutions $\psi(x, t)=e^{-i t} u(x)$ of $(N S P)$ and it contains two kinds of nonlinearities: the first one is $\phi(x) u$ and concerns the interaction with the electric field. This term is nonlocal, since the electrostatic potential $\phi(x)$ depends also on the wave function. The second nonlinearity is $f(u)$. For more information involving physical situations where $(S P)$ appears, we cite the papers of Benci-Fortunato [9], Bokanowski \& Mauser [11, Mauser [24], Ruiz [26], Ambrosetti-Ruiz [4] and S'anchez \& Soler [28].

An important fact involving system $(S P)$ is that this class of system can be transformed into a Schrödinger equation with a nonlocal term (see, for instance, [5, 18, 26, 29]), which allows to use variational methods. Effectively, by the Lax-Milgram Theorem, given $u \in H_{0}^{1}(\Omega)$, there exists a unique $\phi=\phi_{u} \in H_{0}^{1}(\Omega)$ such that

$$
-\Delta \phi=u^{2}
$$

By using standard arguments, we have that $\phi_{u}$ verifies the following properties (for a proof see [15, 26, 29]):

Lemma 1.1 For any $u \in H_{0}^{1}(\Omega)$, we have

i) there exists $C>0$ such that $\left\|\phi_{u}\right\| \leq C\|u\|^{2}$ and

$$
\int_{\Omega}\left|\nabla \phi_{u}\right|^{2} d x=\int_{\Omega} \phi_{u} u^{2} d x \leq C|| u \|^{4} \quad \forall u \in H_{0}^{1}(\Omega)
$$

where $\|u\|^{2}=\int_{\Omega}|\nabla u|^{2} d x$.

ii) $\phi_{u} \geq 0 \forall u \in H_{0}^{1}(\Omega)$;

iii) $\phi_{t u}=t^{2} \phi_{u}, \forall t>0$ and $u \in H_{0}^{1}(\Omega)$; 
iv) if $u_{n} \rightarrow u$ in $H_{0}^{1}(\Omega)$, then $\phi_{u_{n}} \rightarrow \phi_{u}$ in $H_{0}^{1}(\Omega)$ and

$$
\lim _{n \rightarrow+\infty} \int_{\Omega} \phi_{u_{n}} u_{n}^{2} d x=\int_{\Omega} \phi_{u} u^{2} d x .
$$

Therefore, $(u, \phi) \in H_{0}^{1}(\Omega) \times H_{0}^{1}(\Omega)$ is a solution of $(S P)$ if, and only if, $\phi=\phi_{u}$ and $u \in H_{0}^{1}(\Omega)$ is a weak solution of the nonlocal problem

$$
\left\{\begin{array}{l}
-\Delta u+\phi_{u} u=f(u) \text { in } \Omega, \\
u=0, \text { on } \partial \Omega .
\end{array}\right.
$$

Now, we would like to mention that the existence of solutions for problem $(P)$ can be made via variational methods, because if the nonlinearity $f$ belongs to $C^{1}(\mathbb{R}, \mathbb{R})$ and satisfies

$\left(f_{1}\right) \lim _{s \rightarrow 0} \frac{f(s)}{s}=0$;

(f) $\lim _{|s| \rightarrow+\infty} \frac{f(s)}{s^{5}}=0$,

the Lemma 1.1 gives that the functional $J: H_{0}^{1}(\Omega) \rightarrow \mathbb{R}$ given by

$$
J(u)=\frac{1}{2}\|u\|^{2}+\frac{1}{4} \int_{\Omega} \phi_{u} u^{2} d x-\int_{\Omega} F(u) d x,
$$

where

$$
F(s)=\int_{0}^{s} f(t) d t
$$

belongs to $C^{1}\left(H_{0}^{1}(\Omega), \mathbb{R}\right)$ and

$$
J^{\prime}(u) v=\int_{\Omega} \nabla u \nabla v d x+\int_{\Omega} \phi_{u} u v d x-\int_{\Omega} f(u) v d x \quad \forall u, v \in H_{0}^{1}(\Omega) .
$$

Hence, critical points of $J$ are the weak solutions for nonlocal problem $(P)$.

From the above commentaries, we have that system $(S P)$ has a nontrivial solution if, and only if, $(P)$ has a nontrivial solution. This way, in the last years, many authors that studied the system $(S P)$ have focused their attention on problem $(P)$ aiming to establish existence and nonexistence of solutions, multiplicity of solutions, ground state solutions, radial and nonradial solutions, semiclassical limit and concentrations of solution for the 
case where $\Omega=\mathbb{R}^{N}$, see the papers of Azzollini \& Pomponio [5], Cerami \& Vaira [13, Coclite [14, D'Aprile \& Mugnai [15, 16], d'Avenia [17, Ianni [20], Kikuchi [19], and Zhao \& Zhao [29]. For the case where $\Omega$ is a bounded domain, we would like to cite the papers of Siciliano [18], Ruiz \& Siciliano 27] and Pisani \& Siciliano [25]. In all those papers, the solutions found are nonnegative. However, related to nodal ( or sign-changing ) solution, we found few papers, see for example, Ianni [21] and Kim \& Seok [22]. In 21] and 22 the existence of nodal solutions have been established at balls centered origin or in whole $\mathbb{R}^{3}$.

Motivated by papers above, we are interested in finding nodal solution for system $(S P)$, by assuming only that $\Omega \subset \mathbb{R}^{3}$ is a bounded domain with smooth boundary. Once that we will apply variational methods and term $\int_{\Omega} \phi_{u} u^{2} d x$ is homogeneous of degree 4, the corresponding AmbrosettiRabinowitz condition on $f$ is the following:

$(\mathrm{AR})$ There exists $\theta>4$ such that

$$
0<\theta F(s) \leq s f(s) \quad \forall s \in \mathbb{R} \backslash\{0\} .
$$

This condition is important not only to ensure that the functional $J$ has the mountain pass geometry, but also to guarantee that the Palais-Smale, or Cerami, sequences associated with $J$ are bounded. We recall that (AR) implies a weaker condition: there exist $\theta>4$ and $C_{1}, C_{2}>0$ such that

$$
F(s) \geq C_{1}|s|^{\theta}-C_{2}, \quad \forall s \in \mathbb{R} .
$$

However, we consider here another much weaker one, namely,

$\left(f_{3}\right) \lim _{s \rightarrow+\infty} \frac{F(s)}{s^{4}}=+\infty$.

Moreover, we also assume that the nonlinearity $f$ satisfies

$\left(f_{4}\right) \frac{f(s)}{s^{3}}$ is increasing in $|s|>0$.

Remark 1.2 The condition $\left(f_{4}\right)$ implies that $H(s)=s f(s)-4 F(s)$ is a non-negative function, increasing increasing in $|s|$ with

$$
s H^{\prime}(s)=s^{2} f^{\prime}(s)-3 f(s) s>0 \text { for any }|s|>0 .
$$


Our main result is the following

Theorem 1.3 Suppose that $f$ satisfies $\left(f_{1}\right)-\left(f_{4}\right)$. Then problem $(P)$ possesses at least energy nodal solution, which has precisely two nodal domains.

In the proof of Theorem [1.3, we prove that functional $J$ assumes a minimum value on the nodal set

$$
\mathcal{M}=\left\{u \in \mathcal{N}: J^{\prime}(u) u^{+}=J^{\prime}(u) u^{-}=0 \text { and } u^{ \pm} \neq 0\right\}
$$

where $u^{+}=\max \{u(x), 0\}, u^{-}(x)=\min \{u(x), 0\}$ and

$$
\mathcal{N}=\left\{u \in H_{0}^{1}(\Omega) \backslash\{0\}: J^{\prime}(u) u=0\right\} .
$$

More precisely, we prove that there is $w \in \mathcal{M}$ such that

$$
J(w)=\inf _{u \in \mathcal{M}} J(u) .
$$

After, motivated by argument used in Bartsch, Weth \& Willem [6], we use a deformation lemma to prove that $w$ is a critical point of $J$, and so, $w$ is a least energy nodal solution for $(S P)$ with exactly two nodal domains.

Since $J$ has the nonlocal term $\int_{\Omega} \phi_{u} u^{2} d x$, if $u$ is a nodal solution for $J$, we have that

$$
J^{\prime}\left(u^{+}\right) u^{+}=-\int_{\Omega} \phi_{u^{-}}\left(u^{+}\right)^{2}<0 \text { and } J^{\prime}\left(u^{-}\right) u^{-}=-\int_{\Omega} \phi_{u^{+}}\left(u^{-}\right)^{2}<0 .
$$

From this, some arguments used to prove the existence of nodal solutions for problem like

$$
\left\{\begin{array}{l}
-\Delta u=f(u) \text { in } \Omega \\
u=0, \text { on } \partial \Omega
\end{array}\right.
$$

can not be used, and so, a careful analysis is necessary in a lot of estimates, see Section 2 for details.

Before to conclude this introduction, we would like to cite the papers of Alves [1], Alves \& Soares [2, 3], Bartsch, Weth and Willem [6], Bartsch \& Weth [7], Bartsch, Liu \& Weth [8], Castro, Cossio \& Neuberger [12], Zou [30] and their references, where existence of nodal solution has been studied for problem related to $\left(P_{1}\right)$.

The paper is organized as follows. In Section 2, we show some estimates involving functions that change sign, with the most of them being new for problem $(P)$. The Section 3 is devoted to prove the main result Theorem 1.3 . 


\section{$2 \quad$ Important estimates}

In what follows, we denote by $\mathcal{N}$ the Nehari manifold associated with $J$, that is,

$$
\mathcal{N}=\left\{u \in H_{0}^{1}(\Omega) \backslash\{0\}: J^{\prime}(u) u=0\right\} .
$$

A critical point $u_{0} \neq 0$ of $J$ is a ground state of $(P)$ if

$$
J\left(u_{0}\right)=\inf _{\mathcal{N}} J(u)
$$

Since we are looking for least energy nodal solutions (or sign-changing solutions), our goal is to prove the existence of a critical point for $J$ in the set

$$
\mathcal{M}=\left\{u \in \mathcal{N}: J^{\prime}(u) u^{+}=J^{\prime}(u) u^{-}=0 \text { and } u^{ \pm} \neq 0\right\} .
$$

Let us start with some technical lemmas.

Lemma 2.1 There exists $\rho>0$ such that

(i) $J(u) \geq\|u\|^{2} / 4$ and $\|u\| \geq \rho, \forall u \in \mathcal{N}$;

(ii) $\left\|w^{ \pm}\right\| \geq \rho, \forall w \in \mathcal{M}$.

Proof: From $\left(f_{4}\right)$ and Remark 1.2, for any $u \in \mathcal{N}$

$$
4 J(u)=4 J(u)-J^{\prime}(u) u=\|u\|^{2}+\int_{\Omega}[u f(u)-4 F(u)] d x \geq\|u\|^{2}
$$

and so,

$$
J(u) \geq\|u\|^{2} / 4 \forall u \in \mathcal{N} .
$$

From $\left(f_{1}\right)$ and $\left(f_{2}\right)$, there is $C>0$ such that

$$
f(s) s \leq \frac{\lambda_{1}}{2} s^{2}+C s^{6}, \text { for all } s \in \mathbb{R} .
$$

where $\lambda_{1}$ is the first eigenvalue of $\left(-\Delta, H_{0}^{1}(\Omega)\right)$. Since $J^{\prime}(u) u=0$,

$$
\|u\|^{2}<\|u\|^{2}+\int_{\Omega} \phi_{u}|u|^{2} d x=\int_{\Omega} u f(u) d x \leq \frac{\lambda_{1}}{2} \int_{\Omega} u^{2} d x+C \int_{\Omega} u^{6} d x .
$$

Then by Sobolev embeddings,

$$
\|u\|^{2}<\frac{1}{2}\|u\|^{2}+\hat{C}\|u\|^{6}
$$


from where it follows that

$$
\|u\| \geq \rho \forall u \in \mathcal{N}
$$

where $\rho=\left(\frac{1}{2 \hat{C}}\right)^{\frac{1}{4}}$, finishing the proof of $(i)$.

If $w \in \mathcal{M}$, we have that $J^{\prime}(w) w^{ \pm}=0$. Then, a simple computation gives $J^{\prime}\left(w^{ \pm}\right) w^{ \pm}<0$, which implies

$$
\left\|w^{ \pm}\right\|^{2}<\left\|w^{ \pm}\right\|^{2}+\int_{\Omega} \phi_{w^{ \pm}}\left(w^{ \pm}\right)^{2} d x<\int_{\Omega} f\left(w^{ \pm}\right) w^{ \pm} d x .
$$

As in the item $(i)$, we can deduce that $\left\|w^{ \pm}\right\| \geq \rho$.

Lemma 2.2 If $\left(w_{n}\right)$ is a bounded sequence in $\mathcal{M}$ and $p \in(2,6)$, we have

$$
\liminf _{n} \int_{\Omega}\left|w_{n}^{ \pm}\right|^{p} d x>0 .
$$

Proof: From $\left(f_{1}\right)$ and $\left(f_{2}\right)$, given $\varepsilon>0$ there exists $C>0$ such that

$$
f(s) s \leq \varepsilon \lambda_{1} s^{2}+C|s|^{p}+\varepsilon s^{6}, \text { for all } s \in \mathbb{R} .
$$

Since $w_{n} \in \mathcal{M}$, by Lemma 2.1

$\rho^{2} \leq\left\|w_{n}^{ \pm}\right\|^{2}<\int_{\Omega} w_{n}^{+} f\left(w_{n}^{+}\right) d x \leq \varepsilon \lambda_{1} \int_{\Omega}\left(w_{n}^{+}\right)^{2} d x+C \int_{\Omega}\left|w_{n}^{+}\right|^{p} d x+\varepsilon \int_{\Omega}\left(w_{n}^{+}\right)^{6} d x$

that is,

$$
\rho^{2} \leq \varepsilon\left(\lambda_{1} \int_{\Omega}\left(w_{n}^{ \pm}\right)^{2} d x+\int_{\Omega}\left(w_{n}^{ \pm}\right)^{6} d x\right)+C \int_{\Omega}\left|w_{n}^{ \pm}\right|^{p} d x .
$$

Using the boundedness of $\left(w_{n}\right)$, there is $C_{1}$ such that

$$
\rho^{2} \leq \varepsilon C_{1}+C \int_{\Omega}\left|w_{n}^{ \pm}\right|^{p} d x
$$

Fixing $\varepsilon=\frac{\rho^{2}}{2 C_{1}}$, we get

$$
\int_{\Omega}\left|w_{n}^{ \pm}\right|^{p} d x \geq \frac{\rho^{2}}{2 C}
$$

showing that

$$
\liminf _{n} \int_{\Omega}\left|w_{n}^{ \pm}\right|^{p} d x \geq \frac{\rho^{2}}{2 C}>0 .
$$


Lemma 2.3 Let $v \in H_{0}^{1}(\Omega)$ with $v^{ \pm} \neq 0$. Then, there are $t, s>0$ such that $J^{\prime}\left(t v^{+}+s v^{-}\right) v^{+}=0$ and $J^{\prime}\left(t v^{+}+s v^{-}\right) v^{-}=0$.

Proof: It what follows, we consider the vector field

$$
V(s, t)=\left(J^{\prime}\left(t v^{+}+s v^{-}\right)\left(t v^{+}\right), J^{\prime}\left(t v^{+}+s v^{-}\right)\left(s v^{-}\right)\right) .
$$

from $\left(f_{1}\right)-\left(f_{3}\right)$, a straightforward computation yields that there are $0<r<$ $R$ such that

$$
J^{\prime}\left(r v^{+}+s v^{-}\right)\left(r v^{+}\right), \quad J^{\prime}\left(t v^{+}+r v^{-}\right)\left(r v^{-}\right)>0, \forall s, t \in[r, R]
$$

and

$$
J^{\prime}\left(R v^{+}+s v^{-}\right)\left(R v^{+}\right), \quad J^{\prime}\left(t v^{+}+R v^{-}\right)\left(R v^{-}\right)<0, \quad \forall s, t \in[r, R] .
$$

Now, the lemma follows applying Miranda theorem [23].

Hereafter, for $v \in H_{0}^{1}(\Omega)$ with $v^{ \pm} \neq 0$, we consider the functions $h^{v}:[0,+\infty) \times[0,+\infty) \rightarrow \mathbb{R}$ given by

$$
h^{v}(t, s)=J\left(t v^{+}+s v^{-}\right)
$$

and $\Phi^{v}:[0,+\infty) \times[0,+\infty) \rightarrow \mathbb{R}^{2}$ defined as

$$
\Phi^{v}(t, s)=\left(\frac{\partial h^{v}}{\partial t}(t, s), \frac{\partial h^{v}}{\partial s}(t, s)\right)=\left(J^{\prime}\left(t v^{+}+s v^{-}\right) v^{+}, J^{\prime}\left(t v^{+}+s v^{-}\right) v^{-}\right) .
$$

Since $f$ is a $C^{1}$ function, it follows that $\Phi^{v}$ is also a $C^{1}$ map. Moreover, it is easy to check that if $(t, s)$ is a critical point of $h^{v}$, then

$$
\begin{aligned}
h^{v}(t, s)= & h^{v}(t, s)-\frac{1}{4}\left\langle\nabla h^{v}(t, s),(t, s)\right\rangle \\
= & \frac{1}{4} t^{2}\left\|v^{+}\right\|^{2}+\frac{1}{4} \int_{\Omega}\left[f\left(t v^{+}\right) t v^{+}-4 F\left(t v^{+}\right)\right] d x+ \\
& \frac{1}{4} s^{2}\left\|v^{-}\right\|^{2}+\frac{1}{4} \int_{\Omega}\left[f\left(s v^{-}\right) t v^{-}-4 F\left(s v^{-}\right)\right] d x .
\end{aligned}
$$


Lemma 2.4 If $w \in \mathcal{M}$,

(a) $h^{w}(t, s)<h^{w}(1,1)=J(w)$, for all $s, t \geq 0$ such that $(s, t) \neq(1,1)$;

(b) $\operatorname{det}\left(\Phi^{w}\right)^{\prime}(1,1)>0$.

Proof: Once that $w \in \mathcal{M}$, we have $J^{\prime}(w) w^{+}=J^{\prime}(w) w^{-}=0$, and so,

$$
\left\|w^{+}\right\|^{2}+\int_{\Omega} \phi_{w^{+}}\left(w^{+}\right)^{2} d x+\int_{\Omega} \phi_{w^{-}}\left(w^{+}\right)^{2} d x=\int_{\Omega} f\left(w^{+}\right) w^{+} d x
$$

and

$$
\left\|w^{-}\right\|^{2}+\int_{\Omega} \phi_{w^{-}}\left(w^{-}\right)^{2} d x+\int_{\Omega} \phi_{w^{+}}\left(w^{-}\right)^{2} d x=\int_{\Omega} f\left(w^{-}\right) w^{-} d x .
$$

These equalities imply that $(1,1)$ is a critical point of $h^{w}$. On the other hand, condition $\left(f_{3}\right)$ leads to the limit

$$
\lim _{|(t, s)| \rightarrow \infty} h^{w}(t, s)=-\infty
$$

which implies $h^{w}$ assumes a global maximum in some $(a, b)$.

First of all, we claim that $a, b>0$. If $b=0$, we have

$$
J\left(a w^{+}\right) \geq J\left(t w^{+}\right), \quad \forall t>0
$$

and then $J^{\prime}\left(a w^{+}\right)\left(a w^{+}\right)=0$, or equivalently,

$$
a^{2}\left\|w^{+}\right\|^{2}+a^{4} \int_{\Omega} \phi_{w^{+}}\left(w^{+}\right)^{2} d x=\int_{\Omega} f\left(a w^{+}\right) a w^{+} d x .
$$

Since $J^{\prime}\left(w^{+}\right) w^{+}=J^{\prime}(w) w^{+}-\int_{\Omega} \phi_{w^{-}}\left(w^{+}\right)^{2} d x<0$, we derive

$$
\left\|w^{+}\right\|^{2}+\int_{\Omega} \phi_{w^{+}}\left(w^{+}\right)^{2} d x<\int_{\Omega} f\left(w^{+}\right) w^{+} d x
$$

and so,

$$
\left(1-\frac{1}{a^{2}}\right)\left\|w^{+}\right\|^{2}<\int_{\Omega}\left(\frac{f\left(w^{+}\right) w^{+}}{\left(w^{+}\right)^{4}}-\frac{f\left(a w^{+}\right) a w^{+}}{\left(a w^{+}\right)^{4}}\right)\left(w^{+}\right)^{4} d x .
$$


If $a>1$ the left side in this inequality is positive while, from $\left(f_{4}\right)$, the right side is negative. This information gives that $a \leq 1$. Now, combining the Remark 1.2 with the fact that $a \leq 1$, we get

$$
\begin{array}{r}
h^{w}(a, 0)=J\left(a w^{+}\right)=J\left(a w^{+}\right)-\frac{1}{4} J^{\prime}\left(a w^{+}\right)\left(a w^{+}\right)= \\
=\frac{1}{4} a^{2}\left\|w^{+}\right\|^{2}+\frac{1}{4} \int_{\Omega}\left[f\left(a w^{+}\right) a w^{+}-4 F\left(a w^{+}\right)\right] d x \\
\leq \frac{1}{4}\left\|w^{+}\right\|^{2}+\frac{1}{4} \int_{\Omega}\left[f\left(w^{+}\right) w^{+}-4 F\left(w^{+}\right)\right] d x \\
<\frac{1}{4}\left\|w^{+}\right\|^{2}+\frac{1}{4} \int_{\Omega}\left[f\left(w^{+}\right) w^{+}-4 F\left(w^{+}\right)\right] d x+ \\
+\frac{1}{4}\left\|w^{-}\right\|^{2}+\frac{1}{4} \int_{\Omega}\left[f\left(w^{-}\right) w^{-}-4 F\left(w^{-}\right)\right] d x= \\
=J(w)-\frac{1}{4} J^{\prime}(w) w=J(w)=h(1,1)
\end{array}
$$

that is,

$$
h^{w}(a, 0)<h^{w}(1,1)
$$

which is absurd, because $(a, 0)$ is a global maximum point for $h^{w}$. The same type of argument works to show that $a \neq 0$, and the proof of claim is done.

The second claim is $0<a, b \leq 1$. In fact, since $(a, b)$ is another critical point of $h^{w}$,

$$
a^{2}\left\|w^{+}\right\|^{2}+a^{4} \int_{\Omega} \phi_{w^{+}}\left(w^{+}\right)^{2} d x+a^{2} b^{2} \int_{\Omega} \phi_{w^{-}}\left(w^{+}\right)^{2} d x=\int_{\Omega} f\left(a w^{+}\right) a w^{+} d x
$$

and

$$
b^{2}\left\|w^{-}\right\|^{2}+b^{4} \int_{\Omega} \phi_{w^{-}}\left(w^{-}\right)^{2} d x+a^{2} b^{2} \int_{\Omega} \phi_{w^{+}}\left(w^{-}\right)^{2} d x=\int_{\Omega} f\left(b w^{-}\right) b w^{-} d x .
$$

Without loss of generality, we will suppose that $a \geq b$. From this,

$$
a^{2}\left\|w^{+}\right\|^{2}+a^{4} \int_{\Omega} \phi_{w^{+}}\left(w^{+}\right)^{2} d x+a^{4} \int_{\Omega} \phi_{w^{-}}\left(w^{+}\right)^{2} d x \geq \int_{\Omega} f\left(a w^{+}\right) a w^{+} d x
$$

leading to

$$
\left(\frac{1}{a^{2}}-1\right)\left\|w^{+}\right\|^{2} \geq \int_{\Omega}\left(\frac{f\left(a w^{+}\right) a w^{+}}{\left(a w^{+}\right)^{4}}-\frac{f\left(w^{+}\right) w^{+}}{\left(w^{+}\right)^{4}}\right)\left(w^{+}\right)^{4} d x .
$$


If $a>1$ the left side in this inequality is negative, but from $\left(f_{4}\right)$, the right side is positive, thus we can deduce that $a \leq 1$.

To conclude the proof of item $(a)$, we will show that $h^{w}$ does not have global maximum in $[0,1] \times[0,1] \backslash\{(1,1)\}$. From definition of $h^{w}$,

$$
\begin{array}{r}
h^{w}(a, b)=\frac{1}{4} a^{2}\left\|w^{+}\right\|^{2}+\frac{1}{4} \int_{\Omega}\left[f\left(a w^{+}\right) a w^{+}-4 F\left(a w^{+}\right)\right] d x+ \\
\frac{1}{4} b^{2}\left\|w^{-}\right\|^{2}+\frac{1}{4} \int_{\Omega}\left[f\left(b w^{-}\right) b w^{-}-4 F\left(b w^{-}\right)\right] d x .
\end{array}
$$

Then, if $0<a, b \leq 1$ and $(a, b) \neq(1,1)$,

$$
\begin{gathered}
h^{w}(a, b)<\frac{1}{4}\left\|w^{+}\right\|^{2}+\frac{1}{4} \int_{\Omega}\left[f\left(w^{+}\right) w^{+}-4 F\left(w^{+}\right)\right] d x+ \\
\frac{1}{4}\left\|w^{-}\right\|^{2}+\frac{1}{4} \int_{\Omega}\left[f\left(w^{-}\right) w^{-}-4 F\left(w^{-}\right)\right] d x=h^{w}(1,1)
\end{gathered}
$$

showing that,

$$
h^{w}(a, b)<h^{w}(1,1)
$$

and thereby, the proof of item $(a)$ is complete.

The proof of item $(b)$ is the following. By a simple calculation

$$
\operatorname{det}\left(\Phi^{w}\right)^{\prime}(1,1)=G\left(w^{+}\right) G\left(w^{-}\right)-4\left[\int_{\Omega} \phi_{w^{-}}\left(w^{+}\right)^{2} d x\right]^{2}
$$

where

$$
G(v)=\int_{\Omega}\left[f^{\prime}(v) v^{2}-f(v) v\right] d x-2 \int_{\Omega} \phi_{v} v^{2} d x .
$$

From Remark 1.2

$$
G(v) \geq 2\left[\int_{\Omega} f(v) v d x-\int_{\Omega} \phi_{v} v^{2} d x\right] .
$$

Once that

$$
\int_{\Omega} f\left(w^{+}\right) w^{+} d x-\int_{\Omega} \phi_{w^{+}}\left(w^{+}\right)^{2} d x=\left\|w^{+}\right\|^{2}+\int_{\Omega} \phi_{w^{-}}\left(w^{+}\right)^{2} d x
$$

and

$$
\int_{\Omega} \phi_{w^{-}}\left(w^{+}\right)^{2} d x=\int_{\Omega} \phi_{w^{+}}\left(w^{-}\right)^{2} d x
$$


we have that

$$
G\left(w^{+}\right)>2 \int_{\Omega} \phi_{w^{-}}\left(w^{+}\right)^{2} d x
$$

and

$$
G\left(w^{-}\right)>2 \int_{\Omega} \phi_{w^{-}}\left(w^{+}\right)^{2} d x .
$$

Combining the above informations, it follows that $\operatorname{det}\left(\Phi^{w}\right)^{\prime}(1,1)>0$.

Corollary 2.5 Let $v \in H_{0}^{1}(\Omega)$ be a function verifying

$$
v^{ \pm} \neq 0 \text { and } J^{\prime}(v) v^{ \pm} \leq 0 .
$$

Then, there are $t, s \in[0,1]$ such that

$$
t v^{+}+s v^{-} \in \mathcal{M} .
$$

Proof. An immediate consequence of the arguments used in the proof of Lemma 2.4.

\section{$3 \quad$ Existence of least energy nodal solution.}

In this section, our main goal is to prove the Theorem 1.3. In what follows, we denote by $c_{0}$ the infimum of $J$ on $\mathcal{M}$, that is,

$$
c_{0}=\inf _{v \in \mathcal{M}} J(v) .
$$

From Lemma 2.1(i), we deduce that $c_{0}>0$.

Let $\left(w_{n}\right)$ be a sequence in $\mathcal{M}$ such that

$$
\lim _{n} J\left(w_{n}\right)=c_{0} .
$$

Still from Lemma 2.1(i), $\left(w_{n}\right)$ is a bounded sequence. Hence, without loss of generality, we can suppose that there is $w \in H_{0}^{1}(\Omega)$ verifying

$$
\begin{gathered}
w_{n} \rightarrow w \text { in } H_{0}^{1}(\Omega), \\
w_{n} \rightarrow w \text { in } L^{p}(\Omega) \quad \forall p \in\left[1,2^{*}\right)
\end{gathered}
$$


and

$$
w_{n}(x) \rightarrow w(x) \text { a.e. in } \Omega \text {. }
$$

The condition $\left(f_{2}\right)$ combined with the compactness lemma of Strauss [10, Theorem A.I, p.338] gives

$$
\begin{aligned}
\lim _{n} \int_{\Omega}\left|w_{n}^{ \pm}\right|^{p} d x & =\int_{\Omega}\left|w^{ \pm}\right|^{p} d x \\
\lim _{n} \int_{\Omega} w_{n}^{ \pm} f\left(w_{n}^{ \pm}\right) d x & =\int_{\Omega} w^{ \pm} f\left(w^{ \pm}\right) d x
\end{aligned}
$$

and

$$
\lim _{n} \int_{\Omega} F\left(w_{n}^{ \pm}\right) d x=\int_{\Omega} F\left(w^{ \pm}\right) d x
$$

from where it follows together with Lemma 2.2 that $w^{ \pm} \neq 0$. Then, by Lemma 2.3 there are $t, s>0$ verifying

$$
J^{\prime}\left(t w^{+}+s w^{-}\right) w^{+}=0 \text { and } J^{\prime}\left(t w^{+}+s w^{-}\right) w^{-}=0 .
$$

Next, we will show that $t, s \leq 1$. Since $J^{\prime}\left(w_{n}\right) w_{n}^{ \pm}=0$,

$$
\left\|w_{n}^{+}\right\|^{2}+\int_{\Omega} \phi_{w_{n}^{+}}\left(w_{n}^{+}\right)^{2} d x+\int_{\Omega} \phi_{w_{n}^{-}}\left(w_{n}^{+}\right)^{2} d x=\int_{\Omega} f\left(w_{n}^{+}\right) w_{n}^{+} d x
$$

and

$$
\left\|w_{n}^{-}\right\|^{2}+\int_{\Omega} \phi_{w_{n}^{-}}\left(w_{n}^{-}\right)^{2} d x+\int_{\Omega} \phi_{w_{n}^{+}}\left(w_{n}^{-}\right)^{2} d x=\int_{\Omega} f\left(w_{n}^{-}\right) w_{n}^{-} d x .
$$

Taking the limit in the above equalities, we obtain

$$
\left\|w^{+}\right\|^{2}+\int_{\Omega} \phi_{w^{+}}\left(w^{+}\right)^{2} d x+\int_{\Omega} \phi_{w^{-}}\left(w^{+}\right)^{2} d x \leq \int_{\Omega} f\left(w^{+}\right) w^{+} d x
$$

and

$$
\left\|w^{-}\right\|^{2}+\int_{\Omega} \phi_{w^{-}}\left(w^{-}\right)^{2} d x+\int_{\Omega} \phi_{w^{-}}\left(w^{+}\right)^{2} d x \leq \int_{\Omega} f\left(w^{-}\right) w^{-} d x .
$$

Once that

$$
J^{\prime}\left(t w^{+}+s w^{-}\right)\left(t w^{+}\right)=J^{\prime}\left(t w^{+}+s w^{-}\right)\left(s w^{-}\right)=0
$$


it follows that

$$
t^{2}\left\|w^{+}\right\|^{2}+t^{4} \int_{\Omega} \phi_{w^{+}}\left(w^{+}\right)^{2} d x+t^{2} s^{2} \int_{\Omega} \phi_{w^{-}}\left(w^{+}\right)^{2} d x=\int_{\Omega} f\left(t w^{+}\right) t w^{+} d x
$$

and

$$
s^{2}\left\|w^{-}\right\|^{2}+s^{4} \int_{\Omega} \phi_{w^{-}}\left(w^{-}\right)^{2} d x+t^{2} s^{2} \int_{\Omega} \phi_{w^{-}}\left(w^{+}\right)^{2} d x=\int_{\Omega} f\left(s w^{-}\right) s w^{-} d x .
$$

Now, without loss of generality, we will suppose that $s \geq t$. Under this condition,

$$
s^{2}\left\|w^{-}\right\|^{2}+s^{4} \int_{\Omega} \phi_{w^{-}}\left(w^{-}\right)^{2} d x+s^{4} \int_{\Omega} \phi_{w^{-}}\left(w^{+}\right)^{2} d x \geq \int_{\Omega} f\left(s w^{-}\right) s w^{-} d x
$$

and then

$$
\left(\frac{1}{s^{2}}-1\right)\left\|w^{-}\right\|^{2} \geq \int_{\Omega}\left(\frac{f\left(s w^{-}\right) s w^{-}}{\left(s w^{-}\right)^{4}}-\frac{f\left(w^{-}\right) w^{-}}{\left(w^{-}\right)^{4}}\right)\left(w^{-}\right)^{4} d x .
$$

If $s>1$, the left side in this inequality is negative, but from $\left(f_{4}\right)$, the right side is positive, thus we must have $s \leq 1$, which also implies that $t \leq 1$.

Our next step is show that $J\left(t w^{+}+s w^{-}\right)=c_{0}$. Recalling that $t w^{+}+s w^{-} \in \mathcal{M}$, we derive that

$$
c_{0} \leq J\left(t w^{+}+s w^{-}\right)=J\left(t w^{+}+s w^{-}\right)-\frac{1}{4} J^{\prime}\left(t w^{+}+s w^{-}\right)\left(t w^{+}+s w^{-}\right) .
$$

Thus,

$$
c_{0} \leq\left(J\left(t w^{+}\right)-\frac{1}{4} J^{\prime}\left(t w^{+}\right)\left(t w^{+}\right)\right)+\left(J\left(s w^{-}\right)-\frac{1}{4} J^{\prime}\left(s w^{-}\right)\left(s w^{-}\right)\right) .
$$

From Remark 1.2,

$$
J\left(t w^{+}\right)-\frac{1}{4} J^{\prime}\left(t w^{+}\right)\left(t w^{+}\right) \leq J\left(w^{+}\right)-\frac{1}{4} J^{\prime}\left(w^{+}\right)\left(w^{+}\right)
$$

and

$$
J\left(s w^{-}\right)-\frac{1}{4} J^{\prime}\left(s w^{-}\right)\left(s w^{-}\right) \leq J\left(w^{-}\right)-\frac{1}{4} J^{\prime}\left(w^{-}\right)\left(w^{-}\right) .
$$

Hence,

$$
c_{0} \leq\left(J\left(w^{+}\right)-\frac{1}{4} J^{\prime}\left(w^{+}\right)\left(w^{+}\right)\right)+\left(J\left(w^{-}\right)-\frac{1}{4} J^{\prime}\left(w^{-}\right)\left(w^{-}\right)\right) .
$$


Using Fatous' Lemma combined again with Remark 1.2,

$$
c_{0} \leq J\left(t w^{+}+s w^{-}\right) \leq \liminf _{n}\left(J\left(w_{n}\right)-\frac{1}{4} J^{\prime}\left(w_{n}\right) w_{n}\right)=\lim _{n} J\left(w_{n}\right)=c_{0}
$$

from where it follows that

$$
c_{0}=J\left(t w^{+}+s w^{-}\right) .
$$

Until this moment, we have proved that there exists a $w_{o}=t w^{+}+s w^{-} \in$ $\mathcal{M}$, such that $J\left(w_{o}\right)=c_{0}$. In what follows, let us denote $w_{o}$ by $w$, consequently

$$
J(w)=c_{0} \text { and } w \in \mathcal{M} .
$$

To conclude the proof of Theorem 1.3, we claim that $w$ is a critical point for functional $J$. If it is not true, there exist $\alpha>0$ and $v_{0} \in H_{0}^{1}(\Omega)$ with $\left\|v_{0}\right\|=1$ satisfying

$$
J^{\prime}(w) v_{0}=2 \alpha>0 .
$$

Since $J^{\prime}$ is continuous, we fix $r>0$ such that

$$
J^{\prime}(v) v_{0}>\alpha, v^{ \pm} \neq 0, \text { for all } v \in B_{r}(w) \subset H_{0}^{1}(\Omega) .
$$

From now on, fix $D=(\xi, \chi) \times(\xi, \chi) \subset \mathbb{R}^{2}$ with $0<\xi<1<\chi$ such that

(i) $(1,1) \in D$ and $\Phi^{w}(t, s)=0$ in $\bar{D}$ if, and only if, $t=s=1$;

(ii) $c_{0} \notin h^{w}(\partial D)$;

(iii) $\left\{t w^{+}+s w^{-}:(t, s) \in \bar{D}\right\} \subset B_{r}(w)$;

where $h^{w}$ and $\Phi^{w}$ were defined in Lemma 2.4. Since $J$ is continuous, we can fix $r^{\prime}>0$ such that

$$
\mathcal{B}=\overline{B_{r^{\prime}}(w)} \subset B_{r}(w)
$$

and

$$
\mathcal{B} \cap\left\{t w^{+}+s w^{-}:(t, s) \in \partial D\right\}=\emptyset .
$$

Consider the continuous mapping $\rho: H_{0}^{1}(\Omega) \rightarrow[0,+\infty)$, defined by

$$
\rho(u)=\operatorname{dist}\left(u, \mathcal{B}^{c}\right) .
$$

Moreover, set the bounded Lipschitz vector field $V: H_{0}^{1}(\Omega) \rightarrow H_{0}^{1}(\Omega)$ given by

$$
V(u)=-\rho(u) v_{0}
$$


For each $u \in H_{0}^{1}(\Omega)$, we denote by $\eta(\tau)=\eta(\tau, u)$ the unique solution of ODE

$$
\left\{\begin{array}{l}
\eta^{\prime}(\tau)=V(\eta(\tau)), t>0 \\
\eta(0)=u
\end{array}\right.
$$

Observe that

(1) if $u \notin \mathcal{B}, \eta(\tau, u)=u$, for all $t$;

(2) if $u \in \mathcal{B}, \tau \mapsto J(\eta(\tau, u))$ is decreasing and $\eta(\tau, u) \in \mathcal{B}$, for all $\tau>0$;

(3) there exists $\tau_{o}>0$ such that $\left.J(\eta(\tau, w)) \leq J(w)-\left(\left(r^{\prime} \alpha\right) / 2\right)\right) \tau$, for all $0 \leq \tau \leq \tau_{o}$.

The item (1) is an immediate consequence from the definition of $\rho$. The item (2) follows from the inequality

$$
J^{\prime}(\eta(\tau)) \eta^{\prime}(\tau) \leq-\rho(\eta(\tau)) \alpha<0, \quad \forall \eta(\tau) \in \mathcal{B} .
$$

To verify (3), fix $\tau_{o}>0$ such that

$$
\|\eta(\tau, w)-w\| \leq \frac{r^{\prime}}{2}, \text { for all }|\tau| \leq \tau_{o} .
$$

Thus,

$$
\frac{d}{d t} J(\eta(\tau, w)) \leq-\rho(\eta(\tau)) \alpha \leq-\frac{r^{\prime} \alpha}{2} .
$$

Integrating in $\left[0, \tau_{0}\right]$, we have

$$
J\left(\eta\left(\tau_{0}, w\right)\right) \leq J(w)-\frac{r^{\prime} \alpha}{2} \tau_{0} .
$$

Now, consider $\gamma: \bar{D} \rightarrow H_{0}^{1}(\Omega)$ given by.

$$
\gamma(t, s)=\eta\left(\tau_{o}, t w^{+}+s w^{-}\right)
$$

It is easy to see that

$$
\max _{(t, s) \in \bar{D}} J(\gamma(t, s))<c_{0}
$$

because

$$
J(\gamma(t, s)) \leq h^{w}(t, s)<c_{0} \forall(t, s) \in \bar{D} \backslash\{(1,1)\}
$$


and

$$
\left.J(\gamma(1,1)) \leq J(w)-\left(\left(r^{\prime} \alpha\right) / 2\right)\right) \tau_{o}<c_{o}
$$

Consequently $\gamma(\bar{D}) \cap \mathcal{M}=\emptyset$.

On the other hand, setting $\Psi: \bar{D} \rightarrow \mathbb{R}^{2}$ by

$$
\Psi(t, s)=\left(t^{-1} J^{\prime}(\gamma(t, s))\left(\gamma(t, s)^{+}\right), s^{-1} J^{\prime}(\gamma(t, s))\left(\gamma(t, s)^{-}\right)\right)
$$

we derive that

$$
\Psi(t, s)=\left(J^{\prime}\left(t w^{+}+s w^{-}\right) w^{+}, J^{\prime}\left(t w^{+}+s w^{-}\right) w^{-}\right)=\Phi^{w}(t, s) \forall(t, s) \in \partial D .
$$

Then, using the Brouwer's topological degree

$$
d(\Psi, D,(0,0))=d\left(\Phi^{w}, D,(0,0)\right)=\operatorname{sgn}\left(\operatorname{det}\left(\Phi^{w}\right)^{\prime}(1,1)\right)=1
$$

which yields $\Psi$ has a zero $(a, b)$ in $D$. Thereby, there is $(a, b) \in D$ verifying

$$
J^{\prime}(\gamma(a, b))\left(\gamma(a, b)^{ \pm}\right)=0
$$

that is, $\gamma(a, b) \in \mathcal{M}$ which is a contradiction. From this, $w$ is a critical point of $J$, and so, a nodal solution for problem $(P)$. Now, we will show that $w$ has exactly two nodal domains, to this end, we assume by contradiction that

$$
w=u_{1}+u_{2}+u_{3}
$$

with

$$
u_{i} \neq 0, u_{1} \geq 0, u_{2} \leq 0 \text { and } \operatorname{suppt}\left(u_{i}\right) \cap \operatorname{suppt}\left(u_{j}\right)=\emptyset i \neq j(i, j=1,2,3) .
$$

Setting $v=u_{1}+u_{2}$, we see that $v^{ \pm} \neq 0$. Moreover, using the fact that $J^{\prime}(w)=0$, it follows that

$$
J^{\prime}(v)\left(v^{ \pm}\right) \leq 0
$$

By Corollary 2.5, there are $t, s \in(0,1]$ such that

$$
t v^{+}+s v^{-} \in \mathcal{M}
$$

or equivalently,

$$
t u_{1}+s u_{2} \in \mathcal{M}
$$

and so,

$$
J\left(t u_{1}+s u_{2}\right) \geq c_{0} .
$$


On the other hand, repeating the same type of argument explored in the proof of Lemma 2.4 combined with the fact that $u_{3} \neq 0$, we find

$$
J\left(t u_{1}+s u_{2}\right)<J(w)=c_{0},
$$

obtaining a contradiction. This way, $u_{3}=0$, and $w$ has exactly two nodal domains.

Acknowledgments. The authors are grateful to the referees for a number of helpful comments for improvement in this article.

\section{References}

[1] C.O. Alves, Multiplicity of multi-bump type nodal solutions for a class of elliptic problems in $\mathbb{R}^{N}$. Top. Meth. Nonlinear Anal. 34 (2009), 231-250.

[2] C.O. Alves, S.H.M. Soares, On the location and profile of spike-layer nodal solutions to nonlinear Schrödinger equations. J. Math. Anal. Appl. 296 (2004), $563-577$.

[3] C.O. Alves, S.H.M. Soares, Nodal solutions for singularly perturbed equations with critical exponential growth, J. Differential Equations 234 (2007), $464-484$.

[4] A. Ambrosetti \& R. Ruiz, Multiple bound states for the SchrödingerPoisson problem, Commun. Contemp. Math. 10 (2008) 391-404.

[5] A. Azzollini \& A. Pomponio, Ground state solutions for the nonlinear Schrödinger-Maxwell equations, J. Math. Anal. Appl. 345 (2008) 90-108.

[6] T. Bartsch, T. Weth \& M. Willem, Partial symmetry of least energy nodal solution to some variational problems, Journal D'Analyse Mathématique 1 (2005) 1-18

[7] T. Bartsch \& T. Weth, Three nodal solutions of singularly perturbed elliptic equations on domains without topology, Ann. Inst. H. Poincaré Anal. Non Linéaire 22 (2005), 259-281.

[8] T. Bartsch, Z. Liu \& T. Weth, Sign changing solutions of superlinear Schrödinger equations, Comm. Partial Diff. Equations 29 (2004), 25-42. 
[9] V. Benci \& D. Fortunato, An eigenvalue problem for the SchrödingerMaxwell equations, Top. Meth. Nonlinear Anal. 11 (1998) 283-293.

[10] H. Berestycki \& P.L. Lions, Nonlinear scalar field equations, I - existence of a ground state, Arch. Rat. Mech. Analysis, 82, (1983), 313-346.

[11] O. Bokanowski \& N.J. Mauser, Local approximation of the Hartree-Fock exchange potential: a deformation approach, $\mathrm{M}^{3} \mathrm{AS} 9$ (1999), 941-961.

[12] A. Castro, J. Cossio \& J. Neuberger, A sign-changing solution for a superlinear Dirichlet problem, Rocky Mountain Journal of Mathematics. 27, 4 (1997), 1041- 1053.

[13] G. Cerami \& G. Vaira, Positive solutions for some non-autonomous Schrödinger-Poisson systems, J. Differential Equations 248 (2010) 521543.

[14] G.M. Coclite, A multiplicity result for the nonlinear SchrödingerMaxwell equations, Commun. Appl. Anal. 7 (2003) 417-423.

[15] T. D'Aprile \& D. Mugnai, Solitary waves for nonlinear Klein-GordonMaxwell and Schrödinger-Maxwell equations, Proc. Roy. Soc. Edinburgh Sect. A 134 (2004) 893-906.

[16] T. D'Aprile \& D. Mugnai, Non-existence results for the coupled KleinGordon-Maxwell equations, Adv. Nonlinear Stud. 4 (2004) 307-322.

[17] P. d'Avenia, Non-radially symmetric solutions of nonlinear Schrödinger equation coupled with Maxwell equations, Adv. Nonlinear Stud. (2002) 2 177-192.

[18] G. Siciliano, Multiple positive solutions for a Schrödinger-Poisson-Slater system, J. Math. Analysis and Appl. 365 (2010) 288-299.

[19] H. Kikuchi, On the existence of a solution for elliptic system related to the Maxwell-Schrödinger equations, Nonlinear Anal. 67 (2007) 14451456 .

[20] I. Ianni \& G. Vaira, On concentration of positive bound states for the Schrödinger-Poisson problem with potentials, Adv. Nonlinear Stud. 8 (2008) 573-595. 
[21] I. Ianni, Sign-Changing radial solutions for the Schrödinger-PoissonSlater problem, arXiv:1108.2803v1 .

[22] S. Kim \& J. Seok, On nodal solutions of the Nonlinear SchrödingerPoisson equations, Comm. Cont. Math. 14 (2012) 12450041-12450057.

[23] C. Miranda, Un' osservazione su un teorema di Brouwer, Bol. Un. Mat. Ital., 3 (1940) 5-7.

[24] N.J. Mauser, The Schrödinger-Poisson- $X_{\alpha}$ equation, Applied Math. Letters 14 (2001), 759-763.

[25] L. Pisani \& G. Siciliano, Note on a Schrödinger-Poisson system in a bounded domain, Appl. Math. Lett. 21 (2008) 521-528 .

[26] D. Ruiz, The Schrödinger-Poisson equation under the effect of a nonlinear local term, J. Funct. Analysis 237 (2006) 655-674.

[27] D. Ruiz \& G. Siciliano, A note on the Schrödinger-Poisson-Slater equation on bounded domains, Adv. Nonlinear Stud. 8 (2008) 179-190.

[28] O. Sánchez \& J. Soler, Long-time dynamics of the Schrödinger-PoissonSlater system, J. Statistical Physics 114 (2004), 179-204.

[29] F. Zhao \& L. Zhao, Positive solutions for Schrödinger-Poisson equations with a critical exponent, Nonlinear Anal. 70 (2009) 2150-2164.

[30] W. Zou, Sign-Changing critical point theory, Springer, 2008. 